\title{
Numerical Study Using Explicit Multistep Galerkin Finite Element Method for the MRLW Equation
}

\author{
Liquan Mei, ${ }^{1}$ Yali Gao, ${ }^{1}$ Zhangxin Chen $^{2}$ \\ ${ }^{1}$ Department of Computational Science, School of Mathematics and Statistics, \\ Xi'an Jiaotong University, Xi'an 710049, China \\ ${ }^{2}$ Department of Chemical and Petroleum Engineering, Schulich School of Engineering, \\ University of Calgary, Calgary, Alberta, Canada T2N 1N4
}

Received 21 March 2014; accepted 26 January 2015

Published online 6 March 2015 in Wiley Online Library (wileyonlinelibrary.com).

DOI 10.1002/num.21971

In this article, an explicit multistep Galerkin finite element method for the modified regularized long wave equation is studied. The discretization of this equation in space is by linear finite elements, and the time discretization is based on explicit multistep schemes. Stability analysis and error estimates of our numerical scheme are derived. Numerical experiments indicate the validation of the scheme by $L_{2}-$ and $L_{\infty}-$ error norms and three invariants of motion.4 C 2015 Wiley Periodicals, Inc. Numer Methods Partial Differential Eq 31 : $1875-1889,2015$

Keywords: explicit multistep method; finite elements; Galerkin method; modified regularized long wave equation

\section{INTRODUCTION}

Nonlinear partial differential equations are useful in describing a variety of phenomena across a range of disciplines. Most of these equations do not have an analytical solution, or it is extremely difficult and expensive to compute their analytical solutions. Hence the numerical study of these nonlinear partial differential equations is important in practice. The regularized long wave equation is a class of nonlinear evolution equations, which was originally introduced by Peregrine [1] in describing the behavior of the undular bore, and later by Benjamin et al. [2]. This equation plays an important role in describing physical phenomena in various disciplines, such as the nonlinear transverse waves in shallow water, ion-acoustic waves in plasma, magnetohydrodynamics waves in plasma, longitudinal dispersive waves in elastic rods, and pressure waves in liquids gas bubbles. Many numerical methods for the RLW equation have been proposed, which include finite difference methods [3, 4], the Galerkin finite element method [5-8], least square method [9-11],

\footnotetext{
Correspondence to: Liquan Mei, Department of Computational Science, School of Mathematics and Statistics, Xi'an Jiaotong University, Xi'an 710049, China (e-mail: lqmei@mail.xjtu.edu.cn)

Contract grant sponsor: NSF of China; contract grant number: 11371289 and 11371288

Contract grant sponsor: China Scholarship Council; contract grant number: 201206285005
}

(c) 2015 Wiley Periodicals, Inc. 
various collocation methods with quadratic B-splines [12], cubic B-splines [13], septic splines [14], and an explicit multistep method [15].

The RLW equation is a special case of the generalized regularized long wave equation

$$
u_{t}+u_{x}+\delta u^{p} u_{x}-\mu u_{x x t}=0 .
$$

where $\delta$ and $\mu$ are positive constants and $p$ is a positive integer. Some numerical methods [1622] for the GRLW equation have also been presented, such as a finite difference method [16], a decomposition method [18], and a sinc-collation method [21]. Another special case of the GRLW equation is called the modified regularized long wave (MRLW) equation in which $p=2$. Some authors have studied the MRLW equation applying various numerical methods, for example: Gardner et al. [23] using B-spline finite elements, Khalifa et al. [24] using a finite difference method, Khalifa et al. [25] using a collocation method with cubic B-splines, Raslan [26] using the collocation method with quadratic B-spline finite elements and used the central finite difference method for time derivative and Khalifa et al. [27] with the Adomian decomposition method.

In this article, we study the MRLW equation using the linear finite element method in space and explicit multistep method in time. We discuss the stability analysis and error estimates of this method and compare its accuracy with previous studies. Numerical experiments are also provided to verify the validation of the scheme.

The outline of this article is as follows. In the next section, the governing equation, its analytical solution and three invariants are given. The numerical methods including space semidiscretization and fully time space discretization are presented in Section III. Section IV describes the stability analysis and the optimal error estimates are derived in Section V. In Section VI, numerical results are provided to test the theoretical result, and a brief conclusion is drawn in the last section.

\section{GOVERNING EQUATION}

Assuming $\Omega=(a, b)$ to be an open, bounded subset of $R$ and $T>0$ to be a final time, setting $\Omega_{T}=\Omega \times(0, T), J=(0, T]$, we consider the following MRLW equation

$$
\begin{aligned}
u_{t}+u_{x}+6 u^{2} u_{x}-\mu u_{x x t}=0, & \text { in } \Omega_{T}, \\
u=0, & \text { on } \partial \Omega \times[0, T], \\
u=u_{0}, & \text { on } \Omega \times\{t=0\},
\end{aligned}
$$

where $u_{0}: \Omega \rightarrow R$ is the initial condition, $\mu$ is a positive constant and subscripts $x$ and $t$ denote differentiation in space and time, respectively. The physical boundary conditions require $u \rightarrow 0$ as $x \rightarrow \pm \infty$.

The MRLW Eq. (1) has the exact solution as [23]

$$
u(x, t)=\sqrt{c} \operatorname{sech}\left(p\left(x-(c+1) t-x_{0}\right)\right) .
$$

where $p=\sqrt{\frac{c}{\mu(c+1)}}, x_{0}$ and $c$ are arbitrary constants. Furthermore, the MRLW Eq. (1) posses three invariants of motion corresponding to conservation of mass, momentum and energy as [23]

$$
I_{1}=\int_{a}^{b} u d x
$$




$$
\begin{aligned}
& I_{2}=\int_{a}^{b}\left(u^{2}+\mu u_{x}^{2}\right) d x, \\
& I_{3}=\int_{a}^{b}\left(u^{4}-\mu u_{x}^{2}\right) d x .
\end{aligned}
$$

These invariants are used to check the conservative properties of the numerical scheme, especially for problems with no analytical solution and during the collision of solitons.

\section{NUMERICAL METHODS}

Set $V=H_{0}^{1}(\Omega)=\left\{v \in H^{1}(\Omega):\left.v\right|_{\partial \Omega}=0\right\}$. Applying the Green's formula to the problem (1) and using the boundary condition in the definition of $V$, we derive the variational form of problem (1):

$$
\begin{cases}\text { Find } u(x, t): J \rightarrow V, \text { such that } & \\ \left(\frac{\partial u}{\partial t}, v\right)+B(u, v)+C(u, v)+D(u, v)=0, & \forall v \in V, t \in J, \\ (u(x, 0), v)=\left(u_{0}(x), v\right), & \forall v \in V, x \in \Omega,\end{cases}
$$

where

$$
B(u, v)=\int_{a}^{b} \mu u_{x t} \cdot v_{x} d x, \quad C(u, v)=\int_{a}^{b} u_{x} \cdot v d x, \quad D(u, v)=\int_{a}^{b} 6 u^{2} u_{x} \cdot v d x
$$

\section{A. Semidiscretization in Space}

Considering a uniform $1-D$ mesh with mesh size $h=x_{i+1}-x_{i}, i=0,1, \ldots, N-1$, which consists of $N+1$ points $a=x_{0}<x_{1}<\cdots<x_{N-1}<x_{N}=b$, we obtain

$$
\begin{aligned}
& \sum_{i=0}^{N-1}\left(\int_{x_{i}}^{x_{i+1}} \frac{\partial u}{\partial t} \cdot v d x+\int_{x_{i}}^{x_{i+1}} \mu u_{x t} \cdot v_{x} d x\right. \\
& \left.\quad+\int_{x_{i}}^{x_{i+1}} u_{x} \cdot v d x+\int_{x_{i}}^{x_{i+1}} 6 u^{2} u_{x} \cdot v d x\right)=0
\end{aligned}
$$

Using the transformation $x=x_{i}+\eta h, 0 \leq \eta \leq 1, i=0,1, \ldots, N-1$, we derive the following contribution

$$
\int_{0}^{1} \frac{\partial u}{\partial t} \cdot v d \eta+\frac{\mu}{h^{2}} \int_{0}^{1} u_{\eta t} \cdot v_{\eta} d \eta+\frac{1}{h} \int_{0}^{1} u_{\eta} \cdot v d \eta+\frac{6}{h} \int_{0}^{1} u^{2} u_{\eta} \cdot v d \eta=0 .
$$

Define the finite dimension subspace $V_{h} \subset V, V_{h}=\operatorname{span}\left\{L_{1}, L_{2}\right\}$, where

$$
L_{1}=\eta, \quad L_{2}=1-\eta
$$


are linear basis functions on each element. Then the semidiscrete scheme for problem (1) is formulated as follows:

$$
\begin{cases}\text { Find } u_{h}: J \rightarrow V_{h}, \text { such that } & \\ \left(\frac{\partial u_{h}}{\partial t}, v\right)+B\left(u_{h}, v\right)+C\left(u_{h}, v\right)+D\left(u_{h}, v\right)=0, & \forall v \in V_{h}, t \in J, \\ \left(u_{h}(x, 0), v\right)=\left(u_{0}^{h}(x), v\right), & \forall v \in V_{h}, x \in \Omega .\end{cases}
$$

The variation of $u$ over the element $\left[x_{i}, x_{i+1}\right], i=0,1, \ldots, N-1$, is expressed as

$$
u^{e}=\sum_{j=1}^{2} L_{j}(x) u_{j}(t) .
$$

For $j=1,2$, by choosing $v=L_{j}$ in problem (7), an element's contribution is obtained in the form of

$$
\begin{aligned}
& \sum_{i=1}^{2}\left\{\left(\int_{0}^{1} L_{i} \cdot L_{j} d x+\frac{\mu}{h^{2}} \int_{0}^{1} L_{i}^{\prime} \cdot L_{j}^{\prime} d x\right) \frac{\partial u_{i}}{\partial t}\right. \\
& \left.\quad+\frac{1}{h}\left(\int_{0}^{1} L_{i}^{\prime} \cdot L_{j} d x\right) u_{i}+\left(\frac{6}{h} \int_{0}^{1}\left(\sum_{i=1}^{2} u_{i} L_{i}\right)^{2} L_{i}^{\prime} \cdot L_{j} d x\right) u_{i}\right\}=0
\end{aligned}
$$

where the symbol (') denotes differentiation with respect to $\eta$, which, in matrix form, is given by

$$
\left(A^{e}+B^{e}\right) \frac{\partial u^{e}}{\partial t}+C^{e} u^{e}+D^{e}\left(u^{e}\right) u^{e}=0,
$$

where $u^{e}=\left(u_{1}, u_{2}\right)^{T}$ are relevant nodal parameters. The element matrices are

$$
\begin{aligned}
A_{j k}^{e} & =\int_{0}^{1} L_{j} L_{k} d \eta, \\
B_{j k}^{e} & =\frac{\mu}{h^{2}} \int_{0}^{1} L_{j}^{\prime} L_{k}^{\prime} d \eta, \\
C_{j k}^{e} & =\frac{1}{h} \int_{0}^{1} L_{j}^{\prime} L_{k} d \eta, \\
D_{j k}^{e} & =\frac{6}{h} \int_{0}^{1}\left(\sum_{m=1}^{2} u_{m} L_{m}\right)^{2} L_{j}^{\prime} L_{k} d \eta .
\end{aligned}
$$

Assembling contributions from all elements leads to the following matrix equation of the coupled nonlinear ordinary differential equation

$$
(A+B) \frac{\partial u}{\partial t}+C u+D(u) u=0,
$$

where $u=\left(u_{0}, u_{1}, \ldots, u_{N}\right)^{T}$ contains all the nodal parameters. The four assembled matrices are tridiagonal. The general row for each matrix has the following form

$$
A:\left(\frac{1}{6}, \frac{2}{3}, \frac{1}{6}\right)
$$




$$
\begin{aligned}
& B: \frac{\mu}{h^{2}}(-1,2,-1) \\
& C: \frac{1}{2 h}(-1,0,1) \\
& D: \frac{6}{h}\left(-\frac{1}{12}\left(u_{m-1}^{2}+2 u_{m-1} u_{m}+u_{m}^{2}\right),\right. \\
& \left.\frac{1}{12}\left(u_{m-1}^{2}-u_{m+1}^{2}\right)+\frac{1}{6} u_{m}\left(u_{m-1}-u_{m+1}\right), \frac{1}{12}\left(3 u_{m}^{2}+2 u_{m} u_{m+1}+u_{m+1}^{2}\right)\right) .
\end{aligned}
$$

\section{B. Full Discretization}

Let $0=t^{0}<t^{1}<\cdots<t^{M}=T$ be a uniform partition of $J$ into subintervals $J^{n}=\left(t^{n}, t^{n+1}\right), n=$ $0,1, \ldots, M-1$ with length $k=t^{n+1}-t^{n}=\frac{T}{M}$. For a generic function $v$ of time, set $v^{n}=v\left(t^{n}\right)$, we formulate the following linear explicit multistep method

$$
(A+B) \sum_{j=0}^{r} \alpha_{j} u^{n+j}=k \sum_{j=0}^{r-1} \gamma_{j}\left(-C-D\left(u^{n+j}\right)\right) u^{n+j},
$$

where parameter variables $\alpha_{j}$ and $\gamma_{j}$ denote the coefficients of the term $\zeta^{j}$ of the polynomials $\alpha$ and $\gamma$,

$$
\alpha(\zeta)=\sum_{j=1}^{r} \frac{1}{j} \zeta^{r-j}(\zeta-1)^{j}, \gamma(\zeta)=\zeta^{r}-(\zeta-1)^{r} .
$$

Here, we consider the explicit two-step method for the MRLW. For $r=2$, we obtain

$$
\alpha_{0}=\frac{1}{2}, \alpha_{1}=-2, \alpha_{2}=\frac{3}{2}, \gamma_{0}=-1, \gamma_{1}=2,
$$

then we achieve the full discretization scheme for (12)

$$
(A+B)\left(\frac{3}{2} u^{n+2}-2 u^{n+1}+\frac{1}{2} u^{n}\right)=k\left(\left(C+D\left(u^{n}\right)\right) u^{n}-2\left(C+D\left(u^{n+1}\right)\right) u^{n+1}\right) .
$$

We can use the Thomas algorithm to solve the linear algebraic system.

\section{STABILITY ANALYSIS}

In this section, we analyze the linear stability of our scheme to the linearized MRLW equation

$$
u_{t}+\beta u_{x}-\mu u_{x x t}=0
$$

where $\beta=1+6 \max \left|u^{2}\right|$. As in previous works [28], the stability results are obtained by examining the location of the roots of its characteristic polynomial. We use the theory of Schur and von Neumann polynomials and outline the relevant definitions and theorems.

Definition 1. The polynomial $\phi$ is a Schur polynomial if all its roots, $z_{j}$ satisfy $\left|z_{j}\right|<1$. 
Definition 2. The polynomial $\phi$ is a simple von Neumann polynomial if all its roots, $z_{j}$ satisfy $\left|z_{j}\right| \leq 1$ and its roots on unit circle are simple roots.

Definition 3. A numerical scheme is stable if and only if its characteristic polynomial $\phi(z)$ is a simple von Neumann polynomial.

Definition 4. Given the polynomial $\phi(z)=\sum_{j=0}^{n} a_{j} z^{j}$ of degree $N$ with $a_{0}, a_{N} \neq 0$, we define the polynomial

$$
\phi_{1}(z)=\frac{\phi^{*}(0) \phi(z)-\phi(0) \phi^{*}(z)}{z}
$$

where $\phi^{*}(z)=\sum_{j=0}^{n} a_{n-j}^{*} z^{j}$ and $a^{*}$ denotes the complex conjugate of $a$.

Theorem 1. $\phi(z)$ is a simple polynomial if and only if either $|\phi(0)|<\left|\phi^{*}(0)\right|$ and $\phi_{1}(z)$ is a simple von Neumann polynomial or $\phi_{1}(z)$ is identically zero and $\frac{d \phi}{d z}$ is a Schur polynomial.

Applying the discrete Fourier transform $u_{j}=e^{i j \xi h} \tilde{u}$ to Eq. (15), we obtain

$$
M_{1} \frac{d \tilde{u}}{d t}=-i M_{2} \tilde{u}
$$

where $M_{1}=1+2\left(\frac{2 \mu}{h^{2}}-\frac{1}{3}\right) \sin ^{2}\left(\frac{\xi h}{2}\right)$ and $M_{2}=\frac{\beta}{h} \sin (\xi h)$. Exploiting the two-step method (15) for Eq. (18) yields

$$
M_{1}\left(\frac{3}{2} \tilde{u}^{n+2}-2 \tilde{u}^{n+1}+\frac{1}{2} \tilde{u}^{n}\right)+i M_{2} k\left(2 \tilde{u}^{n+1}-\tilde{u}^{n}\right)=0,
$$

and the characteristic polynomial is given by

$$
\phi(z)=M_{1}\left(\frac{3}{2} z^{2}-2 z+\frac{1}{2}\right)+i M_{2} k(2 z-1)
$$

so that $\left|\phi^{*}(0)\right|=|\phi(0)|$ and $\phi_{1}(z) \equiv 0$ hold. In view of the application of Theorem 1 , we consider

$$
\frac{d \phi}{d z}=3 M_{1} z-2 M_{1}+i 2 M_{2} k
$$

and the modulus of its root is

$$
|z|=\frac{2}{3} \sqrt{1+\left(\frac{M_{2}}{M_{1}} \Delta t\right)^{2}}=\frac{2}{3} \sqrt{1+\frac{\beta^{2} k^{2} \sin ^{2}(\xi h)}{h^{2}\left(1+2\left(\frac{2 \mu}{h^{2}}-\frac{1}{3}\right) \sin ^{2}\left(\frac{\xi h}{2}\right)\right)^{2}}},
$$

where $\beta>1$, then we have $|z|<1$.

So from Theorem 1, our explicit two-step numerical scheme is stable. 


\section{ERROR ESTIMATES}

In this section, we shall analyze the optimal error estimates for the fully discrete scheme Eq. (15) in time with explicit two-step scheme.

For $s \in \mathbb{N}$, denote $H^{s}$ the Sobolev space $H^{s}(\Omega), H_{0}^{s}$ the Sobolev space $H_{0}^{s}(\Omega)$ and $\|\cdot\|_{s}$ the usual norm in $H^{s}$. Let $H=L^{2}(\Omega)=H^{0},\|\cdot\|_{0}$ is the $L_{2}$ norm and $\|\cdot\|_{\infty}$ is the $L_{\infty}$ norm.

Consider the explicit two-step method, we can prove that the multistep method satisfies the root condition:

1. If $z_{0}$ is a root of $\alpha$, then $\left\|z_{0}\right\|_{0} \leq 1$,

2. $C_{j}=0, j=0,1,2, C_{3} \neq 0$, with $C_{0}=\alpha(1), C_{i}=\frac{1}{i !}\left(\sum_{j=0}^{2} \alpha_{j} j^{i}-\sum_{j=0}^{1} \gamma_{j} j^{i-1}\right), i \geq 1$.

Let $A: H^{2} \cap H_{0}^{1} \rightarrow H$ and $B: H^{2} \cap H_{0}^{1} \rightarrow L^{2}$ defined by $A v=(I-\mu \triangle) v$ and $B v=\nabla \cdot f(v)$, respectively, where $f(v)=-\left(v+2 v^{3}\right)$. Then $A$ is a linear self-adjoint, positive definite operator and $B$ is a nonlinear operator. Let $V:=D\left(A^{1 / 2}\right)=H_{0}^{1}$ with the norm $\|v\|=\left(\|v\|_{0}^{2}+\mu\|\Delta v\|_{0}^{2}\right)^{1 / 2}$ which is equivalent to $\|\cdot\|_{1}$. Identify $H$ with its dual, let $V^{\prime}$ be the dual of $V$, denote by $(\cdot, \cdot)$ the duality of $V^{\prime}$ and $V$ and by $\|\cdot\|_{\star}$ the dual norm on $V^{\prime}$.

For the previous space discretization, a family of finite dimensional subspaces $\left\{V_{h}\right\}_{0<h \leq 1}$ satisfies the following approximation property:

$$
\inf _{\chi \in V_{h}}\left(\|v-\chi\|_{0}+h\|v-\chi\|_{1}\right) \leq c h^{2}\|v\|_{2}, \forall v \in H^{2} \cap H_{0}^{1} .
$$

We derive the following optimal error estimates.

Theorem 2. Let $\left\{u_{h}^{n}\right\}_{n=0}^{M}$ satisfy the scheme Eq. (15), the solution u of Eq. (1) be sufficiently smooth, and $\left\|u_{h}^{0}-u^{0}\right\|_{0}+\left\|u_{h}^{1}-u^{1}\right\|_{0} \leq C\left(k^{2}+h^{2}\right)$. Then, for h and $k$ sufficiently small, there exits a constant $C$, such that

$$
\max _{0 \leq n \leq M}\left\|u_{h}^{n}-u^{n}\right\|_{0} \leq C\left(k^{2}+h^{2}\right),
$$

where the constant $C$ is independent of $h$ and $k$.

Proof. From [29], we know that we can obtain the result Eq. (24) by proving hypothetical conditions hold in Theorem 4.1 of [29]. We define the bilinear form $a(\cdot, \cdot)$ by

$$
a(v, w)=(v, w)+\mu(\nabla v, \nabla w), \quad \forall v, w \in V,
$$

and use the elliptic projection operator to $V_{h}, R_{h}: V \rightarrow V_{h}$, defined by

$$
a\left(R_{h} v-v, \chi\right)=0, \quad \forall \chi \in V_{h},
$$

this elliptic operator has the following properties:

1. There exists a constant $c$, such that

$$
\left\|v-R_{h} v\right\|_{0} \leq c h\|v\|, \quad \forall v \in V
$$


2. Assuming that $v$ is sufficiently smooth, then

$$
\left\|v-R_{h} v\right\|_{0}+h\left\|v-R_{h} v\right\| \leq c h^{2}\|v\|_{2}, \quad \forall v \in V .
$$

Denoting by $T$ the solution operator of the problem: Given $f \in V^{\prime}$, find $v \in V$, such that

$$
a(v, w)=(f, w), \quad \forall w \in V .
$$

According to the Sobolev inequality, there exists a constant $C_{\star}$, such that

$$
\|v\|_{\infty} \leq C_{\star}\|v\|, \quad \forall v \in H_{0}^{1},
$$

Define the set $\bar{M}$ by $\bar{M}=\{v \in V: \exists t \in J,\|u(\cdot, t)-v\|<1\}$. Now consider the set $\tilde{M}=\left\{u_{h} \in \mathbb{R}: \exists(x, t) \in \Omega \times J,\left\|u(x, t)-u_{h}\right\|_{0}<C_{\star}\right\}$ with $C_{\star}$ the constant in Eq. (28). Obviously, every real function $f \in C^{1}(\mathbb{R} \times J \times \mathbb{R})$ restricted to $\mathbb{R} \times J \times \tilde{M}$ satisfies a Lipschitz condition with Lipschitz constant $L_{f}$; namely,

$$
\|f(\cdot, t, v)-f(\cdot, t, w)\|_{0} \leq L_{f}\|v-w\|_{0}, \quad \forall t \in J, \quad \forall v, w \in \bar{M} .
$$

Thus, one can see that

$$
(B(v)-B(w), \varphi) \leq\|f(v)-f(w)\|_{0} \cdot\|\varphi\| \leq L_{f}\|v-w\|_{0} \cdot\|\varphi\|, \quad \forall v, w \in \bar{M}, \varphi \in V .
$$

Then, for $v, w \in \bar{M}$, we obtain

$$
\begin{aligned}
& \|B(v)-B(w)\|_{\star} \leq L_{1}\|v-w\|, \\
& \|T B(v)-T B(w)\|_{0} \leq L_{2}\|v-w\|_{0} .
\end{aligned}
$$

Define approximations $u_{h}^{n} \in V_{h}$ to $u^{n}, n=2, \ldots, M$, inductively by the scheme

$$
\sum_{j=0}^{2} \alpha_{j}\left[\left(u_{h}^{n+j}, \chi\right)+\mu\left(\nabla u_{h}^{n+j}, \nabla \chi\right)\right]=k \sum_{j=0}^{1} \gamma_{j}\left(\nabla \cdot f\left(u_{h}^{n+j}\right), \chi\right), \quad \forall \chi \in V_{h},
$$

for $n=0,1, \ldots, M-2$. Then, from Theorem 4.1 in [29], for $h$ and $k$ sufficiently small, we have

$$
\max _{0 \leq n \leq M}\left\|u_{h}^{n}-u^{n}\right\|_{0} \leq C\left(k^{2}+h^{2}\right)
$$

with a constant $C$ independent of $h$ and $k$.

\section{NUMERICAL EXPERIMENTS}

In this section, we present some numerical tests to check the efficiency and accuracy of our explicit two-step scheme, which are the propagation of single soliton and collision of two solitons at different time levels. Finally, we investigate the development of the Maxwellian initial condition into solitary waves. 
TABLE I. Invariants and error norms for single solitary wave, $c=1.0, h=0.2, k=0.025, x_{0}=40$, and $0 \leq x$ $\leq 100$.

\begin{tabular}{lccccc}
\hline Time & $I_{1}$ & $I_{2}$ & $I_{3}$ & $L_{2}$ & $L_{\infty}$ \\
\hline Analytical & 4.44288 & 3.29983 & 1.41421 & & \\
0 & 4.44288 & 3.29981 & 1.41420 & 0 & 0 \\
2 & 4.44288 & 3.29981 & 1.41421 & $2.38994 \times 10^{-3}$ & $1.23221 \times 10^{-3}$ \\
4 & 4.44288 & 3.29982 & 1.41423 & $4.56503 \times 10^{-3}$ & $2.36119 \times 10^{-3}$ \\
6 & 4.44288 & 3.29982 & 1.41421 & $6.49892 \times 10^{-3}$ & $3.42251 \times 10^{-3}$ \\
8 & 4.44288 & 3.29983 & 1.41421 & $8.14728 \times 10^{-3}$ & $4.32579 \times 10^{-3}$ \\
10 & 4.44288 & 3.29983 & 1.41420 & $9.49238 \times 10^{-3}$ & $5.06983 \times 10^{-3}$ \\
\hline
\end{tabular}

To illustrate the accuracy of the present scheme, we use $L_{2}-$ and $L_{\infty}-$ error norms

$$
\begin{aligned}
& L_{2}=\left\|u_{h}^{n}-u^{n}\right\|_{0}=\sqrt{\left(h \sum_{i=1}^{N}\left|u_{h j}^{n}-u_{j}^{n}\right|^{2}\right)}, \\
& L_{\infty}=\left\|u_{h}^{n}-u^{n}\right\|_{\infty}=\max _{j}\left|u_{h j}^{n}-u_{j}^{n}\right| .
\end{aligned}
$$

to compare the numerical solution with the exact solution, which shows the mean and maximum differences between the numerical and the analytical solutions. The quantities $I_{1}, I_{2}$, and $I_{3}$ measure the conservation laws of our scheme during propagation.

\section{A. Single Soliton}

The analytical values of the variants are

$$
I_{1}=\frac{\pi \sqrt{c}}{p}, I_{2}=\frac{2 c}{p}+\frac{2 \mu p c}{3}, I_{3}=\frac{4 c^{2}}{3 p}-\frac{2 \mu p c}{3} .
$$

For the purpose of comparison with previous works [23] and [25], we choose the parameters $x_{0}=40, c=1.0, h=0.2, k=0.025, T=10$, and $0 \leq x \leq 100$. Then the amplitude is 1.0 and the analytical values of the three invariants are $I_{1}=4.44288, I_{2}=3.29983$, and $I_{3}=1.41421$. The relevant numerical results are presented in Table I, and the profiles of solitary waves at different time are given in Fig. 1. Table II presents the variants, $L_{\infty}$ norm and $L_{2}$ norm at time $=10$ against the cubic B-splines in [23] and [25].

To illustrate the accuracy of our algorithm, we compute the pointwise convergence rate in time and space. We define the order of accuracy of space as the formulation [7]

$$
\operatorname{order}_{h}=\frac{\log _{10}\left(\left|u-u_{h_{j}}\right| /\left|u-u_{h_{j+1}}\right|\right)}{\log _{10}\left(h_{j} / h_{j+1}\right)},
$$

where $|\cdot|$ denotes $L_{2}$ or $L_{\infty}$ norm, and $u_{h_{j}}$ is the numerical solution with spatial step size $h_{j}$. Fixing the temporal step size $k=0.01$, we choose the parameters as $c=0.03$ and different spatial partition $0.2,0.4$, and 0.8 . The $L_{2}-$ and $L_{\infty}$ - error and the convergence order of time are presented in Tables III and IV. We can obtain $L_{2}-$ and $L_{\infty}-$ error norms

$$
\left\|u_{h}-u\right\|_{0} \approx 0.036 h^{2.026},\left\|u_{h}-u\right\|_{\infty} \approx 0.01886 h^{2.008}
$$

by linear regression, reach $O\left(h^{2}\right)$ and confirm the theoretical results. 


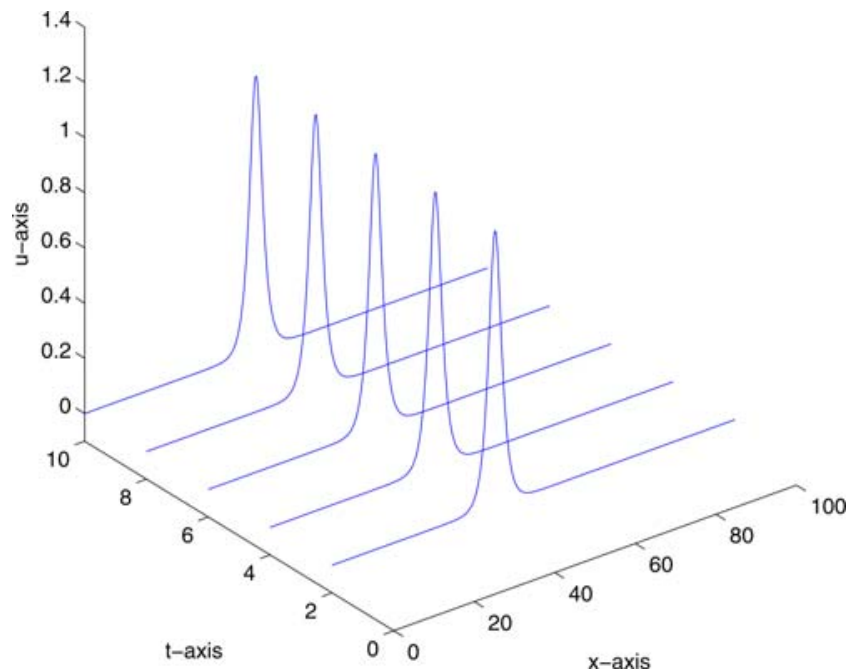

FIG. 1. Solitary wave with $c=1.0, h=0.2, k=0.025$, and $x_{0}=40,0 \leq x \leq 100$. [Color figure can be viewed in the online issue, which is available at wileyonlinelibrary.com.]

TABLE II. Invariants and error norms for single solitary wave, $c=1.0, h=0.2, k=0.025, x_{0}=40,0 \leq x \leq$ 100 , and time $=10$.

\begin{tabular}{llllll}
\hline Time & \multicolumn{1}{c}{$I_{1}$} & \multicolumn{1}{c}{$I_{2}$} & \multicolumn{1}{c}{$I_{3}$} & \multicolumn{1}{c}{$L_{2}$} & $L_{\infty}$ \\
\hline Analytical & 4.44288 & 3.29983 & 1.41421 & & \\
Our scheme & 4.44288 & 3.29983 & 1.41420 & $9.49238 \times 10^{-3}$ & $5.06983 \times 10^{-3}$ \\
B-splines [25] & 4.44288 & 3.29983 & 1.41420 & $9.30196 \times 10^{-3}$ & $5.43718 \times 10^{-3}$ \\
B-splines coll-CN [23] & 4.442 & 3.299 & 1.413 & $1.639 \times 10^{-2}$ & $9.24 \times 10^{-3}$ \\
B-splines coll+PA-CN [23] & 4.440 & 3.296 & 1.411 & $2.03 \times 10^{-2}$ & $1.12 \times 10^{-2}$ \\
\hline
\end{tabular}

TABLE III. The order of convergence of space for $L_{2}$ error norm with $c=0.3$ and $k=0.01$.

\begin{tabular}{lccccc}
\hline Time & $h=0.2$ & $h=0.4$ & $h=0.8$ & Order $(0.2 / 0.4)$ & Order $(0.4 / 0.8)$ \\
\hline 2 & $5.19056 \times 10^{-4}$ & $2.21847 \times 10^{-3}$ & $9.30613 \times 10^{-3}$ & 2.09559 & 2.06862 \\
4 & $9.63368 \times 10^{-4}$ & $4.09489 \times 10^{-3}$ & $1.68473 \times 10^{-2}$ & 2.08767 & 2.04062 \\
6 & $1.35709 \times 10^{-3}$ & $5.74562 \times 10^{-3}$ & $2.33937 \times 10^{-2}$ & 2.08194 & 2.02559 \\
8 & $1.72854 \times 10^{-3}$ & $7.29675 \times 10^{-3}$ & $2.95259 \times 10^{-2}$ & 2.07770 & 2.01665 \\
10 & $2.08976 \times 10^{-3}$ & $8.80161 \times 10^{-3}$ & $3.54682 \times 10^{-2}$ & 2.07443 & 2.01069 \\
\hline
\end{tabular}

TABLE IV. The order of convergence of space for $L_{\infty}$ error norm with $c=0.3$ and $k=0.01$.

\begin{tabular}{lccccc}
\hline Time & $h=0.2$ & $h=0.4$ & $h=0.8$ & Order $(0.2 / 0.4)$ & Order $(0.4 / 0.8)$ \\
\hline 2 & $3.41517 \times 10^{-4}$ & $1.45341 \times 10^{-3}$ & $6.17747 \times 10^{-3}$ & 2.08941 & 2.08758 \\
4 & $5.60435 \times 10^{-4}$ & $2.38594 \times 10^{-3}$ & $9.76806 \times 10^{-3}$ & 2.08994 & 2.03351 \\
6 & $7.24409 \times 10^{-4}$ & $3.07040 \times 10^{-3}$ & $1.25052 \times 10^{-3}$ & 2.08355 & 2.02602 \\
8 & $8.77790 \times 10^{-4}$ & $3.70389 \times 10^{-3}$ & $1.48963 \times 10^{-2}$ & 2.07709 & 2.00784 \\
10 & $1.02762 \times 10^{-3}$ & $4.34071 \times 10^{-3}$ & $1.69394 \times 10^{-2}$ & 2.07862 & 1.96438 \\
\hline
\end{tabular}


TABLE V. The order of convergence of time for $L_{2}$ error norm with $c=0.3$ and $h=0.125$.

\begin{tabular}{lccccc}
\hline Time & $k=0.05$ & $k=0.1$ & $k=0.2$ & Order $(0.05 / 0.1)$ & Order $(0.1 / 0.2)$ \\
\hline 2 & $8.10432 \times 10^{-4}$ & $3.76761 \times 10^{-3}$ & $1.49388 \times 10^{-2}$ & 2.21688 & 1.96567 \\
4 & $1.52224 \times 10^{-3}$ & $7.25869 \times 10^{-3}$ & $3.07186 \times 10^{-2}$ & 2.25352 & 2.06015 \\
6 & $2.11029 \times 10^{-3}$ & $1.02249 \times 10^{-2}$ & $4.48009 \times 10^{-2}$ & 2.27658 & 2.11015 \\
8 & $2.62859 \times 10^{-3}$ & $1.29309 \times 10^{-2}$ & $5.84011 \times 10^{-2}$ & 2.29847 & 2.15365 \\
10 & $3.10977 \times 10^{-3}$ & $1.55296 \times 10^{-2}$ & $7.22347 \times 10^{-2}$ & 2.32014 & 2.19589 \\
\hline
\end{tabular}

TABLE VI. The order of convergence of time for $L_{\infty}$ error norm with $c=0.3$ and $h=0.125$.

\begin{tabular}{lccccc}
\hline Time & $k=0.05$ & $k=0.1$ & $k=0.2$ & Order $(0.05 / 0.1)$ & Order $(0.1 / 0.2)$ \\
\hline 2 & $5.29688 \times 10^{-4}$ & $2.46747 \times 10^{-3}$ & $9.73878 \times 10^{-3}$ & 2.21982 & 1.96567 \\
4 & $8.87783 \times 10^{-4}$ & $4.23136 \times 10^{-3}$ & $1.77624 \times 10^{-2}$ & 2.25284 & 2.06015 \\
6 & $1.13236 \times 10^{-3}$ & $5.47313 \times 10^{-3}$ & $2.36326 \times 10^{-2}$ & 2.27304 & 2.11015 \\
8 & $1.34030 \times 10^{-3}$ & $6.55669 \times 10^{-3}$ & $2.90663 \times 10^{-2}$ & 2.29042 & 2.15365 \\
10 & $1.53374 \times 10^{-3}$ & $7.59687 \times 10^{-3}$ & $3.45147 \times 10^{-2}$ & 2.30835 & 2.19589 \\
\hline
\end{tabular}

Similar to the definition of the spatial order accuracy, the convergence rate of time can be defined as

$$
\operatorname{order}_{t}=\frac{\log _{10}\left(\left|u-u_{k_{j}}\right| /\left|u-u_{k_{j+1}}\right|\right)}{\log _{10}\left(k_{j} / k_{j+1}\right)},
$$

where $u_{k_{j}}$ is the numerical solution with time step size $k_{j}$. Tables V and VI propose the $L_{2}$ and $L_{\infty}$ error convergence rate of time with $c=0.3$, the space size $h=0.125$ and the time size $k=$ $0.05,0.2,0.4$. We have

$$
\left\|u_{h}-u\right\|_{0} \approx 1.439 k^{2.164},\left\|u_{h}-u\right\|_{\infty} \approx 0.7033 k^{2.127} .
$$

which reach $O\left(k^{2}\right)$ in accordance with the theoretical analysis.

\section{B. Collision of Two Solitons}

In this section, we study the interaction of two solitary waves with initial conditions given by a linear sum of two separate solitary waves of various amplitudes

$$
u(x, 0)=\sum_{i=1}^{2} A_{i} \operatorname{sech}\left(p_{i}\left(x-x_{i}\right)\right),
$$

where $A_{i}=\sqrt{c_{i}}, p_{i}=\sqrt{\frac{c_{i}}{\mu\left(c_{i}+1\right)}}, i=1,2, x_{i}$ and $c_{i}(i=1,2)$ are arbitrary constants. The analytical values of the conservation laws in this case have the following form

$$
\begin{aligned}
& I_{1}=\frac{\pi \sqrt{c_{1}}}{p_{1}}+\frac{\pi \sqrt{c_{2}}}{p_{2}}, \\
& I_{2}=\frac{2 c_{1}}{p_{1}}+\frac{2 c_{2}}{p_{2}}+\frac{2 \mu c_{1} p_{1}}{3}+\frac{2 \mu p_{2} c_{2}}{3},
\end{aligned}
$$

Numerical Methods for Partial Differential Equations DOI 10.1002/num 
TABLE VII. Invariants for two solitary waves with $c_{1}=1, c_{2}=0.5, x_{1}=20, x_{2}=30, h=0.1, k=0.01$, and $0 \leq x \leq 100$.

\begin{tabular}{lccc}
\hline Time & $I_{1}$ & $I_{2}$ & $I_{3}$ \\
\hline 0 & 8.29053 & 5.29563 & 1.88406 \\
2 & 8.29054 & 5.29562 & 1.88387 \\
4 & 8.29054 & 5.29557 & 1.88364 \\
6 & 8.29054 & 5.29550 & 1.88333 \\
8 & 8.29055 & 5.29545 & 1.88306 \\
10 & 8.29055 & 5.29545 & 1.88309 \\
\hline
\end{tabular}

$$
I_{3}=\frac{4 c_{1}^{2}}{3 p_{1}}+\frac{4 c_{2}^{2}}{3 p_{2}}-\frac{2 \mu p_{1} c_{1}}{3}-\frac{2 \mu p_{2} c_{2}}{3} .
$$

In our numerical scheme, we choose $h=0.1, k=0.01, c_{1}=1.0, c_{2}=0.5, x_{1}=20$, and $x_{2}=30$ with interval $[0,100]$, then the amplitudes are in ratio $\sqrt{2}: 1$, where $\sqrt{2} A_{2}=A_{1}$ and the analytical values for the conservations are $I_{1}=8.29053, I_{2}=5.22433$, and $I_{3}=1.79911$. The changes of the invariants are satisfactorily small, as the changes of the invariants $I_{1}, I_{2}$, and $I_{3}$ are $2.41239 \times 10^{-6}, 3.39903 \times 10^{-5}$, and $5.14845 \times 10^{-4}$, respectively. The results are recorded in Table VII. The geometry of initial state and profiles at time $t=0,3,7$, and 10 shown graphically in Fig. 2, respectively.

\section{The Maxwellian Initial Condition}

Now, we consider the development of the Maxwellian initial condition

$$
u(x, 0)=e^{-(x-40)^{2}},
$$

into a train of solitary waves. We choose different values $\mu=1.0$ and 0.5 in our numerical scheme. The comparisons of the three variants $I_{1}, I_{2}$, and $I_{3}$ with the earlier result in [30] are presented in Table VIII. For $\mu=1.0$, the change of the variants $I_{2}$ and $I_{3}$ with respect to the initial values are $6.1624 \times 10^{-4}$ and $8.5756 \times 10^{-3}$, respectively, and the change of the variants $I_{1}$ from the initial variants approaches zeros. For $\mu=0.5$, the variants are changed by $0,4.2646 \times 10^{-5}$ and $5.9698 \times 10^{-3}$ in this case. The development of the Maxwellian initial condition is shown in Fig. 3 with different parameter values, respectively. The smaller $\mu$ is, the more solitary waves will form. The simulations are done up to time $=10$ in this case. From the previous work [23], the total number of solitary waves and the values $\mu$ has the approximate relation

$$
N \cong\left[\frac{1}{\sqrt[5]{\mu}}\right]
$$

\section{CONCLUSION}

In this article, the explicit mutistep Galerkin finite element method is taken to investigate the propagation of nonlinear partial differential equation of the MRLW equation. The stability analysis is given, and optimal error estimate proves that the convergence of our scheme can reach $O\left(k^{2}+h^{2}\right)$. The numerical results in Tables III-VI show that the $L_{2}-$ and $L_{\infty}$ - error norms are of order $O\left(k^{2}+h^{2}\right)$, corresponding with theoretical results. The high efficiency and accuracy of 

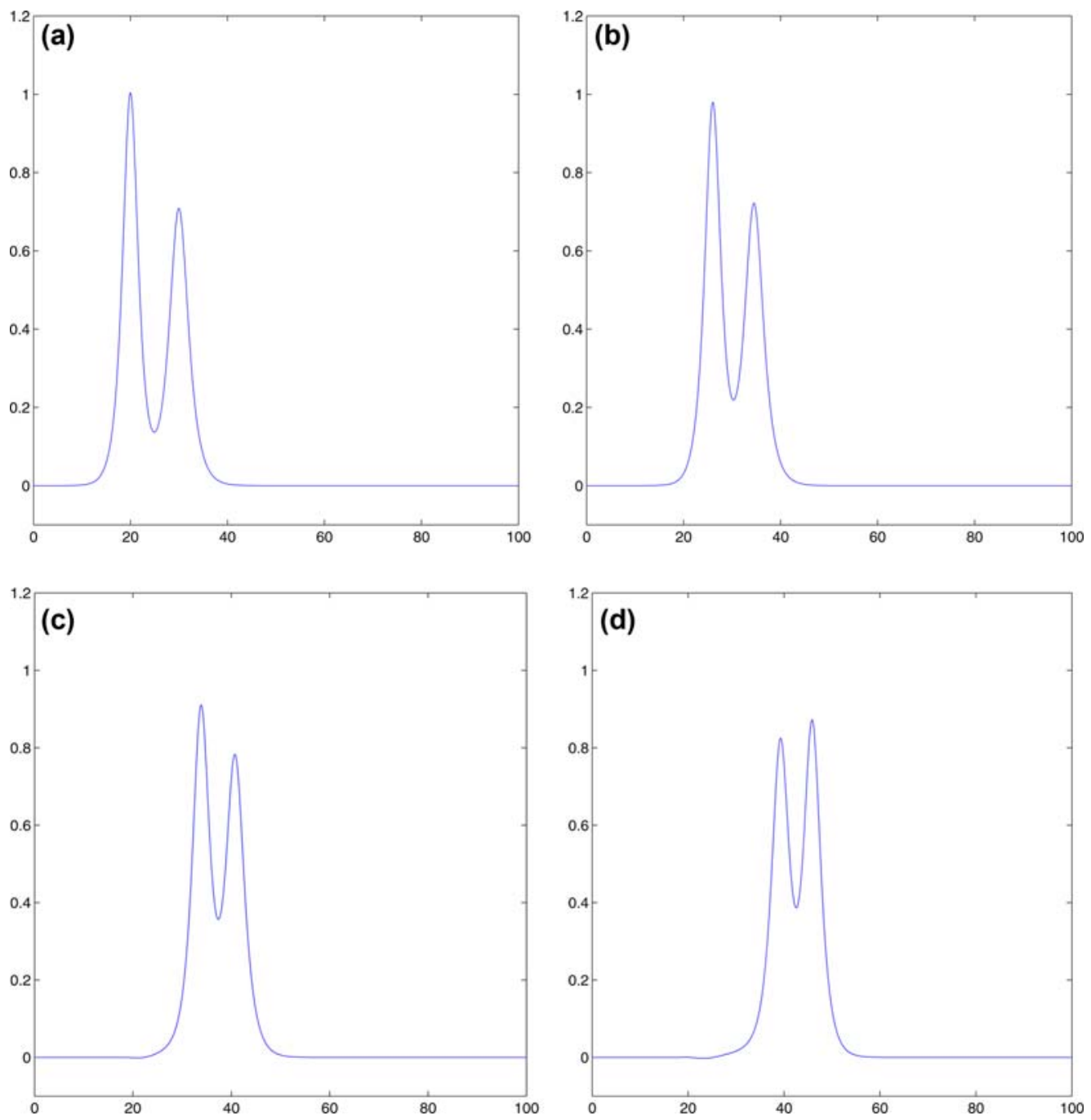

FIG. 2. Interaction of two solitary waves. [Color figure can be viewed in the online issue, which is available at wileyonlinelibrary.com.]

TABLE VIII. Values of $I_{1}, I_{2}, I_{3}$ for Maxwellian initial condition when $h=0.1, k=0.01$, and the space interval $[0,100]$.

\begin{tabular}{|c|c|c|c|c|c|c|c|}
\hline$\mu$ & Time & $I_{1}$ & $I_{2}$ & $I_{3}$ & $I_{1}[30]$ & $I_{2}[30]$ & $I_{3}[30]$ \\
\hline \multirow{4}{*}{1} & 2 & 1.77254 & 2.49904 & -0.363239 & 1.77245 & 2.50635 & -0.366815 \\
\hline & 4 & 1.77254 & 2.49904 & -0.364212 & 1.77245 & 2.50624 & -0.366697 \\
\hline & 6 & 1.77254 & 2.49934 & -0.365635 & 1.77245 & 2.50617 & -0.366633 \\
\hline & 8 & 1.77254 & 2.50037 & -0.365984 & 1.77245 & 2.50612 & -0.366586 \\
\hline \multirow{6}{*}{0.5} & 10 & 1.77254 & 2.50058 & -0.366354 & 1.77244 & 2.50609 & -0.366555 \\
\hline & 2 & 1.77254 & 1.87592 & 0.259807 & 1.77245 & 1.87989 & 0.259650 \\
\hline & 4 & 1.77254 & 1.87585 & 0.258868 & 1.77245 & 1.87986 & 0.259683 \\
\hline & 6 & 1.77254 & 1.87588 & 0.258465 & 1.77245 & 1.87984 & 0.259697 \\
\hline & 8 & 1.77254 & 1.87585 & 0.258335 & 1.77245 & 1.87983 & 0.259705 \\
\hline & 10 & 1.77254 & 1.87584 & 0.258256 & 1.77245 & 1.87983 & 0.259709 \\
\hline
\end{tabular}



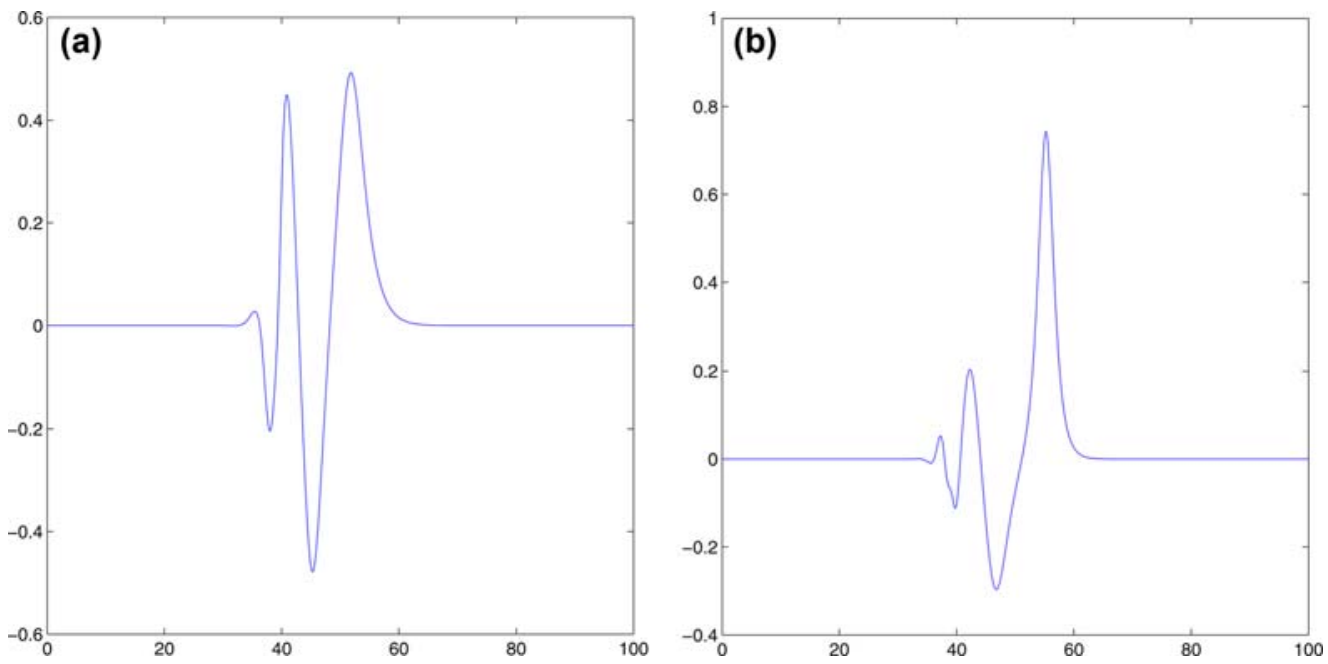

FIG. 3. Maxwellian initial condition with different values of $\mu$. [Color figure can be viewed in the online issue, which is available at wileyonlinelibrary.com.]

our numerical method are demonstrated by the numerical experiments: the propagation of single solitons, collision of two and the development of Maxwellian initial condition into solitary waves. Moreover, our scheme satisfies the three conservation laws of mass, momentum and energy.

\section{References}

1. D. H. Peregrine, Calculations of the development of an undular bore, J Fluid Mech 25 (1966), 321-330.

2. T. B. Benjamin, J. L. Bona, and J. J. Mahony, Model equation for long wave in non-linear dispersive systems, Philos Trans R Soc London A 272 (1972), 47-78.

3. S. Kutluay and A. Esen, A finite difference solution of the regularized longwave equation, Math Probl Eng 2006 (2006), 1-14.

4. J. Lin, Z. Xie, and J. Zhou, High-order compact difference scheme for the regularized long wave equation, Commun Numer Methods Eng 23 (2007), 135-156.

5. P. Avilez-Valente, F. J. Seabra-Santos, and A Petrov-Galerkin, Finite element scheme for the regularized long wave equation, Comput Mech 34 (2004), 256-270.

6. A. Esen and S. Kutluay, Application of a lumped Galerkin method to the regularized long wave equation, Appl Math Comput 174 (2006), 833-845.

7. L. Mei and H. Chen, Numerical solutons of MRLW equation using Glalerkin method with extraplation techniques, Comput Phys Commun 183 (2012), 1609-1616.

8. B. Saka and I. Dag, A numerical solution of the RLW equation by Galerkin method using quartic B-splines, Commun Numer Methods Eng 24 (2008), 1339-1361.

9. I. Dag, Least squares quadratic B-spline finite element method for the regularized long wave equation, Comput Methods Appl Mech Eng 182 (2000), 205-215.

10. I. Dag and M. N. Ozer, Approximation of the RLW equation by the least square cubic B-spline finite element method, Appl Math Model 25 (2001), 221-231.

11. H. Gu, N. Chen, Least-squares mixed finite element methods for the RLW equations, Numer Methods Partial Differential Equations 24 (2008), 749-758. 
12. A. A. Soliman and K. R. Raslan. Collocation method using quadratic B-spline for the RLW equation, Int J Comput Math 78 (2001), 399-412.

13. I. Dag, B. Saka, and D. Irk, Application of cubic B-splines for numerical solution of the RLW equation, Appl Math Comput 159 (2004), 373-389.

14. A. A. Soliman and M. H. Hussien, Collocation solution for RLW equation with septic spline, Appl Math Comput 161 (2005), 623-636.

15. L. Mei and Y. Chen, Explicite multistep method for the numerical solution of RLW equation, Appl Math Comput 218 (2012), 9547-9554.

16. L. Zhang, A finite difference scheme for generalized long-wave equation, Appl Math Comput 168 (2005), 962-972

17. M. Mohammadi and R. Mokhtari, Solving the generalized regularized long wave equation on the basis of a reproducing kernel space, J Comput Appl Math 235 (2011), 4003-4014.

18. D. Kaya, A numerical simulation of solitary-wave solutions of the generalized regularized long-wave equation, Appl Math Comput 149 (2004), 833-841.

19. T. Roshan and A. Petrov-Galerkin method for solving the generalized regularized long wave (GRLW) equation, Comput Math Appl 63 (2012), 943-956.

20. A. A. Soliman, Numerical simulation of the generalized regularized long wave equation by He's variational iteration method, Math Comput Simul 70 (2005), 119-124.

21. R. Mokhtari and M. Mohammadi, Numerical solution of GRLW equation using Sinc-collocation method, Comput Phys Commun 181 (2010), 1266-1274.

22. A. H. A. Ali, A. A. Soliman, and K. R. Raslan, Soliton solution for nonlinear partial differential equations by cosine-function method, Phys Lett A 368 (2007), 299-304

23. L. R. T. Gardner, G. A. Gardner, F. A. Irk, and N. K. Amein, Approximation of solitary wave of the MRLW equation by B-spline finite element, Arab J Sci Eng 22 (1997), 183-193.

24. A. K. Khalifa, K. R. Raslan, and H. M. Alzubaidi, A finite difference scheme for the MRLW and solitary wave interactions, Appl Math Comput 189 (2007), 346-354.

25. A. K. Khalifa, K. R. Raslan, and H. M. Alzubaidi, A collocation method with cubic B-splines for solving the MRLW equation, J Comput Appl Math 212 (2008), 406-418.

26. K. R. Raslan, Numerical study of the Modified Regularized Long Wave (MRLW) equation, Chaos Solitions Fractals 42 (2009), 1845-1853.

27. A. K. Khalifa, K.R. Raslan, and H.M. Alzubaidi, Numerical study using ADM for the modified regularized long wave equation, Appl Math Model 32 (2008), 2962-2972.

28. U. M. Ascher, S.J. Ruuth, and B. T. R. Wetton, Implicit-explicit methods for time dependent partial differential equations, SIAM J Numer Anal 32 (1995), 797.

29. P. Chatzipantelidis, Explicit multistep methods for nonstiff partial differential equations, Appl Numer Math 27 (1998), 13-31.

30. K. R. Raslan and T. S. EL-Danaf, Solitary waves sollutions of the MRLW equation using quintic B-splines, J King Saud University 22 (2010), 161-166. 